OPEN ACCESS

Edited by:

Silvia Giordani,

Dublin City University, Ireland

Reviewed by:

Francisco M. Raymo,

University of Miami, United States

Stefania Impellizzeri,

Ryerson University, Canada

${ }^{*}$ Correspondence:

Guglielmo Lanzan

guglielmo.lanzani@iit.it

Chiara Bertarelli

chiara.bertarelli@polimi.it

${ }^{+}$These authors have contributed

equally to this work.

Specialty section:

This article was submitted to

Smart Materials,

a section of the journal

Frontiers in Materials

Received: 20 November 2020

Accepted: 18 December 2020

Published: 21 January 2021

Citation:

Vurro V, Bondelli G, Sesti V, Lodola F, Paternò GM, Lanzani G and Bertarelli C (2021) Molecular Design of Amphiphilic

Plasma Membrane-Targeted

Azobenzenes for

Nongenetic Optical Stimulation.

Front. Mater. 7:631567.

doi: 10.3389/fmats.2020.631567

\section{Molecular Design of Amphiphilic Plasma Membrane-Targeted Azobenzenes for Nongenetic Optical Stimulation}

\author{
Vito Vurro ${ }^{1,2 \dagger}$, Gaia Bondelli ${ }^{1,2 \dagger}$, Valentina Sesti $i^{2,3}$, Francesco Lodola ${ }^{2,4}$, \\ Giuseppe Maria Paternò ${ }^{2}$, Guglielmo Lanzani ${ }^{1,2 *}$ and Chiara Bertarelli ${ }^{2,3 *}$
}

${ }^{1}$ Department of Physics, Politecnico di Milano, Milan, Italy, ${ }^{2}$ Center for Nano Science and Technology @PoliMi, Istituto Italiano di Tecnologia, Milan, Italy, ${ }^{3}$ Department of Chemistry, Politecnico di Milano, Milan, Italy, ${ }^{4}$ Department of Biotechnology and Biosciences, Università di Milano-Bicocca, Milan, Italy

We present a series of cationic membrane-targeted azobenzene molecules, with the aim to understand how variations in molecular architecture influence the relative optical and biological properties. 1,4-Amino-substituted azobenzene was chosen as the switching unit while the number of linked alkyl chains and their cationic end-group were systematically varied. Their photophysics, membrane partitioning, and electrophysiological efficacy were studied. We found that the polar end group is a keyfactor determining the interaction with the phospholipid heads in the plasma membrane bilayer and consequently the ability to dimerize. The monosubstituted photoswitch with a pyridinium-terminated alkyl chain was found to be the best candidate for photostimulation. This study provides a structure-property investigation that can guide the chemical engineering of a new generation of molecular photo-actuators.

Keywords: photochromic molecules, photostimulation, cell membrane, amphiphilic compounds, spectroscopy

\section{INTRODUCTION}

In the field of bio-stimulation, light allows clean, precise, and nondestructive control and manipulation of a wide range of biological effects. Compared with electrically driven stimulation methods, optical techniques lead to better performances in terms of spatio-temporal resolution, efficiency, and fast response, providing unprecedented opportunities in neuroscience, chemical biology, and photopharmacology (Scanziani and Häusser, 2009; Chae et al., 2010; Bareket-Keren and Hanein, 2014; Fang et al., 2020). Standard bio-targets such as eukaryotic cells are not intrinsically responsive to light due to their transparency. Nevertheless, the development of optogenetics successfully provided a tool to modulate the biological activity through the expression of exogenous light-sensitive proteins (Fenno et al., 2011). Another useful approach consists in the use of photoactive materials attached to the bio-target, enabling the conversion of light excitation into electrical, chemical, mechanical, or thermal response (Di Maria et al., 2018; Hopkins et al., 2019; Fang et al., 2020). In these regards, the use of optical switches introduces additional opportunities in photostimulation, as the cell sensitivity can be reversibly modulated depending on the molecules spatial arrangement (Uchida, 2004; Russew and Hecht, 2010). Within this context, azobenzenes are able to repeatedly and reversibly switch their molecular configuration between two isomeric forms upon irradiation at a specific wavelength range. The relatively high photostability of azobenzenes, as well as the easiness of its chemical functionalization, have enabled a wide use as a reversible 
photoactuator in a range of applications, spanning from photopharmacology (Beharry and Woolley, 2011; Velema et al., 2014; Tochitsky et al., 2016; Fuchter, 2020) to drug delivery (Geng et al., 2017). More specifically, the optical and biological responses of such molecules can be greatly modified by groups and structures attached to the azobenzene unit, giving the possibility to tailor azobenzene derivatives with optimized membrane internalization, thermal stability, and tuned light absorption range (Griffiths, 1972; Crecca and Roitberg, 2006; Bandara and Burdette, 2012). In our previous works, we reported a newly synthesized amphiphilic azobenzene molecule named ZIAPIN2 (DiFrancesco et al., 2020; Paternò et al., 2020b), designed to be amphiphilic in order to drive spontaneous partitioning into the cell membrane. This was due to the presence of a polar terminal group able to interact with the phospholipidic heads of the membrane bilayer and a hydrophobic moiety that can stabilize inside the bilayer lipidic region. ZIAPIN2 has proven to work as an intramembrane light actuator that is capable to modulate neuronal firing in vitro as well as in vivo by virtue of an opto-mechanical effect (DiFrancesco et al., 2020). In particular, the trans isomer causes a thinning of the membrane possibly by forming membrane-spanning dimers, whereas light illumination leads to the breaking of these dimers, causing a relaxation of the membrane thickness. Such an effect translates into a photomodulation (increase/decrease) of the cell membrane capacitance and, hence, to a light-triggered electrophysiological response.

Motivated by these results, here we report the design of a series of cationic membrane-targeted photoswitches based on azobenzene, taking ZIAPIN2 architecture as a reference. We tested their optical and biological efficacy examining their photophysics, membrane partitioning, and electrophysiology, focusing on the media the molecules are dissolved in, as the molecules local microenvironment potently affects their spectral features (Paternò et al., 2020b). Indeed, despite our proof of concept studies were based on the widely used dimethyl sulfoxide (DMSO) as vehicle solvent, here we selected water in order to exclude any possible influence of DMSO on the cell properties [e.g., membrane stiffness (Paternò et al., 2020a)] and to enhance the potential impact in biological applications. In addition, the azobenzene derivatives were suspended in a solution of 1palmitoyl-2-oleoyl-glycero-3-phosphocholine

(POPC) liposomes, which mimic the cellular lipid bilayer environment.

\section{RESULTS AND DISCUSSION UV-Vis and Photoluminescence Measurements}

The set of azo-compounds herein proposed can be divided into three categories: 1) aze-pyridinium (Pyr $\left.\left.\left[\mathrm{C}_{5} \mathrm{H}_{5} \mathrm{NH}\right]^{+}\right)\right]$) compounds containing an azepane as one terminal and one or more pyridinium as polar head (following this new notation, the previously presented ZIAPIN2 takes the name Az-2Pyr); 2) azeammonium compounds, with trimethylammonium (TMA, $\left.\mathrm{N}\left(\mathrm{CH}_{3}\right)_{3}\right)^{+}$cationic end group as polar head instead of pyridinium; 3) cross compounds, whose azobenzene rings are fully substituted with four Pyr- or TMA-terminated alkyl chains. The last class represents a symmetric switch, which in principle can interact with the phosphate heads of both membrane leaflets hence identified as a transmembrane molecule. Figure $1 \mathrm{~A}$ reports the molecular structures of the six different azobenzene molecules, highlighting the three categories into three different columns. Sharing the same azobenzene core structure, the molecules are named using the following labels identifying the side featuring groups: Az, Pyr, and TMA which indicate azepane, pyridinium, and trimethylammonium, respectively, whereas the number represents how many substituted alkyl chains are present on one and the other side of azobenzene (i.e., only on one side for $\mathrm{Az}$ compounds). For all the investigated compounds, we firstly evaluated the affinity for the membrane environment by means of absorption/photoluminescence spectroscopy and confocal microscopy, followed by the investigation on their isomerization ability and electrophysiological effects.

We used UV-Vis absorption and photoluminescence (PL) spectroscopies to examine the behavior of the six photoswitches in water (Figures 1B, C) and POPC liposomes (Figures 1D,E). Reference data of the molecules dispersed in DMSO as a control medium and vector are reported in Supplementary Figure S1. All compounds in DMSO show the typical UV-Vis absorption features of para di-amino-substituted azobenzenes, with a strong absorption structured band centered at $470 \mathrm{~nm}$, which can be ascribed to the superimposed $S_{0} \rightarrow S_{2}$ and red-shifted $S_{0} \rightarrow S_{1}$ transitions of the trans isomer. On the other hand, in water, the molecules exhibit featureless and redshifted absorption and PL spectra in comparison with the linewidth observed in DMSO (Supplementary Figure S2), as it has been already reported for Az-2Pyr (Paternò et al., 2020b). This effect has been associated to the formation of molecular aggregates (excimers) that hinders isomerization favoring radiative deactivation. In POPC liposome suspension, we note that the absorption and PL bands lie in between water and DMSO. In addition, for both the 2-chains molecules Az-2Pyr and Az-2TMA, the absorbance spectra are strongly structured, presenting three different vibronic peaks, which can be related to the incorporation of the molecules in the lipid bilayer. In this context, having attributed the red-shifted and featureless absorption and PL bands to the presence of nonisomerizing molecular aggregates (Paternò et al., 2020b), the blue-shift observed in POPC liposomes indicates a partial reacquisition of the isomerization pathway. This, however, does not apply for the two cross molecules 2Pyr-2Pyr and 2TMA-2TMA, whose PL spectra remain peaked around $620 \mathrm{~nm}$. Moreover, the doublepeaked PL spectrum of $2 \mathrm{Pyr}-2 \mathrm{Pyr}$ suggests to be given by the superimposition of at least two contributions, one at $580 \mathrm{~nm}$ due to the fluorescence of the cross molecule intercalated inside the liposomes and one peaking at around $610 \mathrm{~nm}$ due to molecular aggregates formed in the aqueous environment. Based on these data, we expect an appreciable isomerization dynamic only for those derivatives presenting small red shifts in water solution, namely, 1- and 2-chains molecules in POPC. Note that in general the number of substituents on the central azobenzene ring system seems to drive the shift in both absorption and PL shown in 


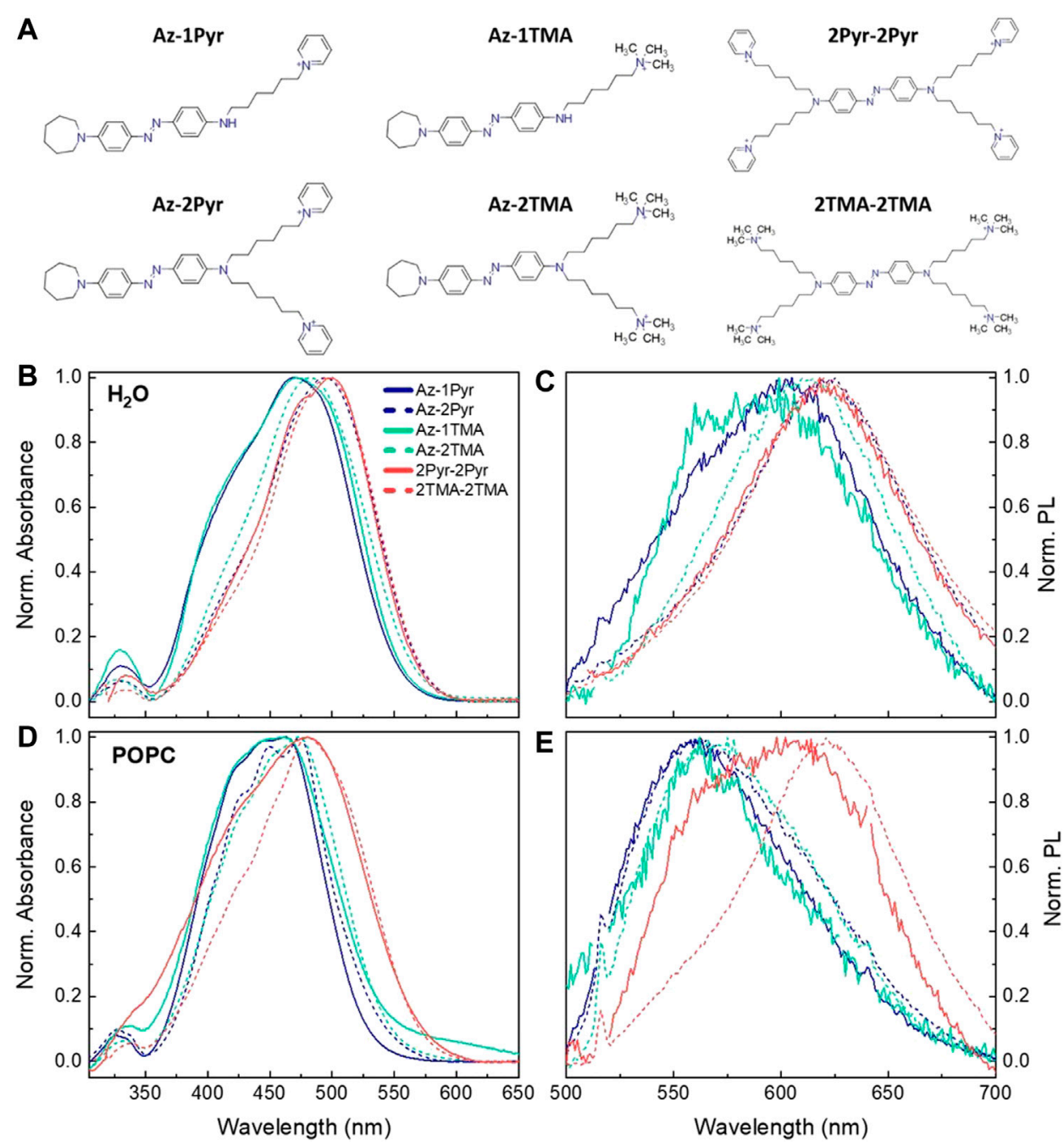

FIGURE 1 | (A) Chemical formulas of the six designed azobenzene derivatives. UV-Vis and PL spectra in water (B, C) and in POPC liposome suspension (D, E). PL spectra have been acquired with fixed excitation at $470 \mathrm{~nm}$ and were normalized to both lamp intensity and ground state absorption. A scattering background profile has been subtracted from the POPC absorbance spectra.

Figure 1: the higher the number of side alkyl chains, the larger the red-shift (Bandara and Burdette, 2012).

\section{Membrane Partitioning}

Having established the intrinsic affinity of these photoswitches for the phospholipidic environment of artificial liposomes, we assessed the localization and the relative partitioning efficacy inside cell membrane through fluorescence confocal imaging. In vitro tests were performed incubating separately each compound into glass-plated HEK-293 cells, widely used in biotechnology as the experimental model because of their reliable and rapid growth rate. In order to evaluate the affinity of azobenzenes for the plasma membrane, the colocalization between the studied molecules and a living cells membrane marker was measured. Z-stack images (Figure 2) were acquired in at least three independent experiments, and the relative colocalization was evaluated using methods based on Pearson's coefficient (Bolte and Cordelières, 2006). The estimated overlap coefficient (OC) reaches a good value for all the six molecules, evidencing their preference for the membrane environment rather than for their initial aggregate state in the aqueous vehicle solvent (Figure 2D). All molecules indeed show a mean OC value higher than $66 \%$. In particular, mean OC values for the monosubstituted molecules are around $83 \%$ for Az-1Pyr and 66\% for Az-1TMA, while for their bisubstituted equivalents Az-2Pyr and Az-2TMA, OC values are around $78 \%$ and $81 \%$, respectively. For the highly symmetric cross molecules 2Pyr-2Pyr and 2TMA-2TMA, this parameter reaches $74 \%$ and $77 \%$, respectively.

We have to point out that during this measurements, a reproducibility issue emerged with the monoterminated ammino-derivative, Az-1TMA, which we attributed to the difficulty to solubilize this molecule in water. Despite this, we still report Az-1TMA data along this work. From OC values, it emerges that the lower the number of end-groups, the higher the localization into cell membrane. Moreover, TMA molecules show better membrane partitioning with respect to their $\mathrm{Pyr}$ 


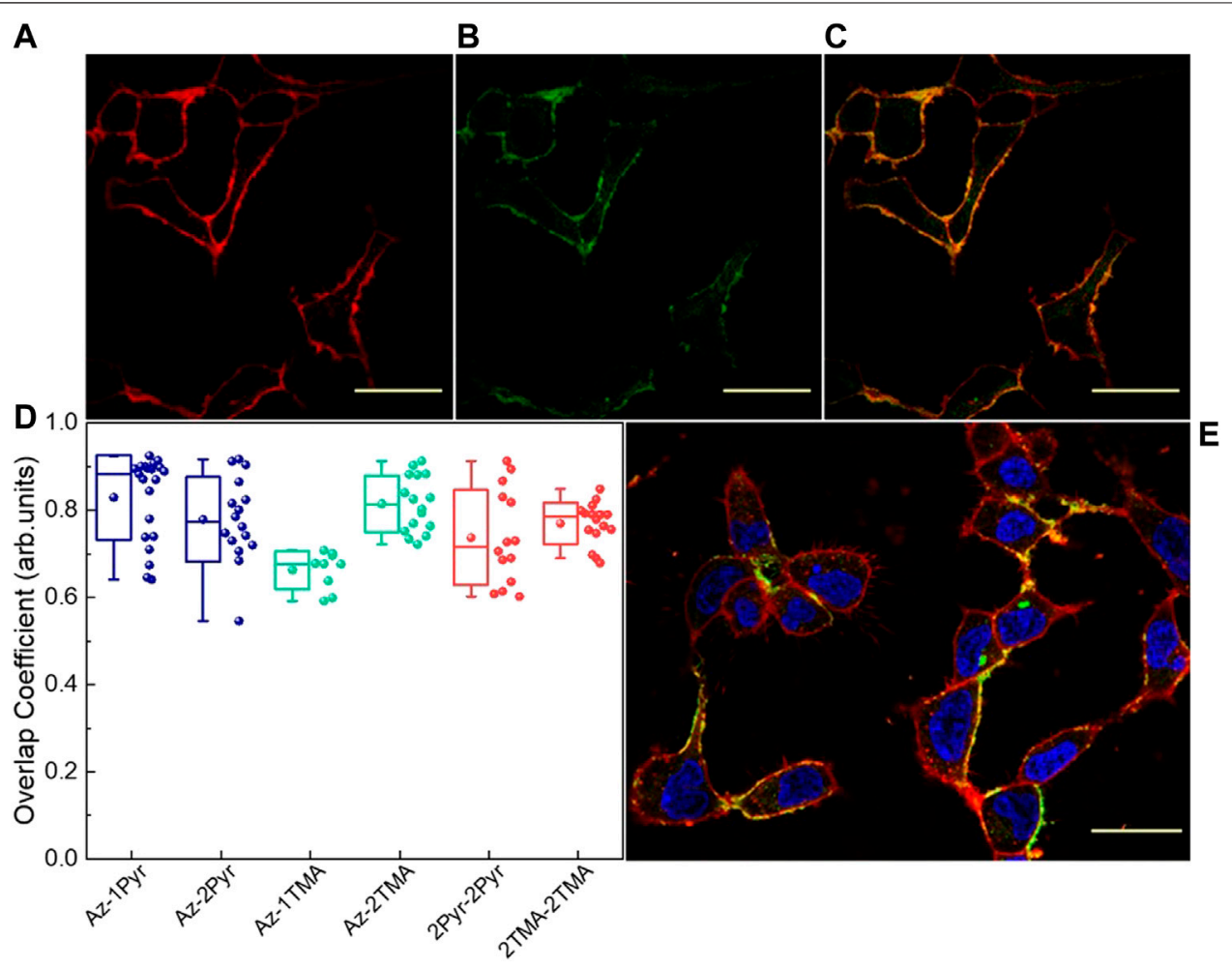

FIGURE 2 | Fluorescence confocal images showing the Cell Mask labeled HEK-293 cell membranes (A), Az-2Pyr molecule (25 $\mu$ M) (B), and their colocalization (C), 7 min after incubation and rinsing. (D) Colocalization analysis overlap coefficient (OC) values for the six molecules ( $n \geq 10)$. (E) HEK-293 cells treated with Az-2Pyr and stained with CellMask (red) and DAPI (blue). Scale bar $=50 \mu \mathrm{m}$.

counterparts, exception made for Az-1TMA. We speculate that the average lower OC values shown by the two cross compounds are due to an intrinsic stronger affinity for the aggregate state. Alternatively, lower OC values can be due to the absence of the azepane moiety in their structure. In fact, this group has higher solubility in the aliphatic part of the membrane due to its low polarity, which could promote the molecules dwelling in the membrane.

Possible changes in the OC values can occur due to molecules affinity for specific membrane micro domains [lipid rafts, as previously reported (DiFrancesco et al., 2020)]. To consider this, we also evaluated Mander's overlap coefficients (MOCs), M1 and M2 (Supplementary Figure S3). MOC parameters evaluate the colocalization of the first images pixel with the nonzero intensity pixel of the second image (M1) and vice versa (M2). In this way, with $\mathrm{M} 2$, it is possible to find out any possible affinity for specific domains of the membrane. MOC parameters confirm what came out with the use of OC, particularly highlighting the behavior of Az-1Pyr and Az-2Pyr. Despite good localization, however, a higher data dispersion is present, indicating a strong dependency on the efficacy of the partitioning process, which can greatly vary from sample to sample.

\section{Collective Isomerization Dynamics}

Before investigating the electrophysiological behavior triggered by the molecules, we assessed the photodynamic ability of each compound by means of UV-Vis spectroscopy. Absorption spectra of a $25 \mu \mathrm{M}$ solution of each compound in water and POPC were measured under illumination with a blue LED $(470 \mathrm{~nm})$, monitoring the variation of peak intensities at two wavelengths: 360 and $450 \mathrm{~nm}$, which are indicative of the cis and trans isomer form, respectively. For the latter case, we monitored the absorption at $450 \mathrm{~nm}$ rather than at the absorption maximum $(470 \mathrm{~nm})$, to reduce artefacts given by the scattered excitation light. Measurements in water (Supplementary Figure S4) show no signatures of isomerization, in agreement with what already observed in our previous investigations (Paternò et al., 2020b). Therefore, these data confirmed that water suppresses azobenzene photoisomerization due to the formation of aggregates hindering molecular switching. Conversely, the isomerization ability is recovered when the molecules are internalized in liposomes, confirming that the membrane environment enables the photoisomerization of the amphiphilic azobenzenes. For instance, Supplementary Figure S5 shows the spectral changes of Az-2Pyr in POPC upon light irradiation, with direct trans $\rightarrow$ cis isomerization recognizable by the decrease of the $470 \mathrm{~nm}$ absorption band and the contextual rise of the 360 and $550 \mathrm{~nm}$ bands, which are characteristic of the cis isomer absorption. We present here the isomerization kinetics of our azobenzene series in POPC liposome suspension taken at the two selected wavelengths as a function of illumination time. Looking at the kinetics at $450 \mathrm{~nm}$, the abrupt steps in the decay profiles of 2Pyr-2Pyr and 2TMA-2TMA (Supplementary Figure S6) can be almost solely associated with scattering from the 

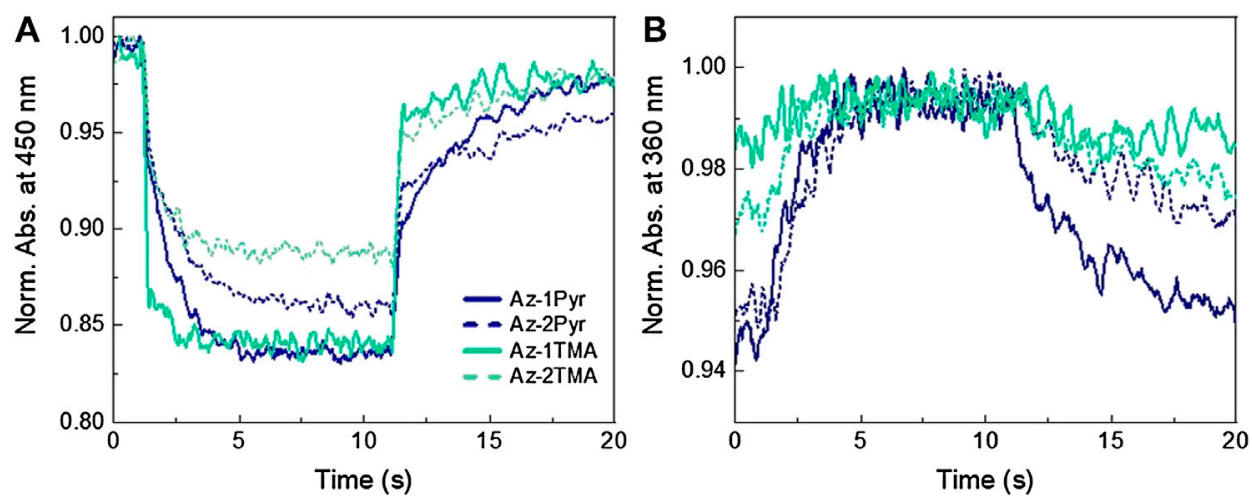

FIGURE 3 | Isomerization kinetics of the azobenzene series in POPC liposomes taken at $450 \mathrm{~nm}$ (A) and $360 \mathrm{~nm}$ (B) as a function of illumination time. Data were taken through steady-state absorption measurements of the molecules in solution under illumination with a blue LED (470 nm).

excitation lamp, while the exponential decays in the other compounds are in-fact due to trans $\rightarrow$ cis isomerization reaction (Figure 3A). This is even more evident looking at the rise of the cis isomer peak $(360 \mathrm{~nm})$, whose spectral region is not affected by the scattering of the lamp (Figure 3B). Here, we can see that Az-1Pyr and Az-2Pyr and, to less extent, Az-1TMA and Az-2TMA exhibit an increase in the cis isomer population. Specifically, the amount of trans isomer converting to the cis form is $24 \%$ for Az-1Pyr, $21 \%$ for Az-2Pyr, $17 \%$ for Az-1TMA, and $19 \%$ for Az-2TMA. Again, we have to specify that the water solubility issue mentioned before affects data acquired for Az1TMA. For what regards the two tetrasubstituted molecules (2TMA-2TMA and 2Pyr-2Pyr), from their data we can infer that they both do not undergo effective isomerization in the lipid membrane environment, probably due to the formation of strongly bound aggregates in the lipid membrane. The redshifted Abs/PL of the cross molecules reported in Figure 1 and the colocalization analysis reported in Figure 2 corroborate this scenario. The collective isomerization dynamics of the molecules were best fitted with a single exponential function (see Supplementary Table S1 for the time constants). Interestingly, we note a correlation between molecular polar end-groups and time constants, as the isomerization process is accelerated by the presence of the azeammonium compounds. Considering the dimerization hypothesis mentioned in our introduction, we can speculate that once partitioned in the membrane, Az-TMA molecules form weaker trans-dimers than the Az-Pyr azobenzenes, which can be disrupted easily and quickly by light irradiation. Moreover, the TMA termination influences the amphiphilic character of the molecule, thereby affecting the aggregation tendencies of both trans and cis isomers. Cis conformation could also be destabilized, with respect to the trans one, through steric and electrostatic repulsion. An even more complex relationship is instead present between number of attached polar heads and time constant. Indeed, compounds synthesized with two side-chains show slower isomerization than the single-substituted ones. Finally, the fact that the monosubstituted species (smaller dipole moment, "less amphiphilic") relaxes more quickly than the disubstituted one (larger dipole moment, "more amphiphilic") is a sign of the role of increased amphiphilicity inside the azeammonium class, where it could promote aggregation again in very well agreement with the localization analysis.

\section{Membrane Potential Modulation}

Finally, HEK-293 cells loaded with the compounds were investigated with whole-cell patch-clamp recordings in order to evaluate quantitatively the membrane modulation action extent upon compound photoisomerization. In these experiments, short $(20 \mathrm{~ms})$ and long $(200 \mathrm{~ms})$ light stimuli were administered to the cells, separately loaded with each compound (Supplementary Figure S7). The one by one hyperpolarization/depolarization effect, already reported for Az-2Pyr (DiFrancesco et al., 2020; Paternò et al., 2020b), was observed for all the azobenzene molecules, with the exception of $2 \mathrm{Pyr}-2 \mathrm{Pyr}$, whose nonphotoreactivity was already noted from photoisomerization measurements in artificial membranes. Furthermore, we can observe a correlation between polar head group and biological effect, as the aze-pyridinium molecules lead to a stronger perturbation in the hyperpolarization phase with respect to ammonium-substituted photoswitches. On the other hand, the lower dispersion of the depolarization values confirms the nature of this process, which is in fact related to a balancing process actuated by cells and is not related to the photoreaction, as previously reported (Hodgkin and Huxley, 1952; DiFrancesco et al., 2020). Such a membrane potential variation is induced by modulation of the membrane electrical capacitance triggered by a decrease in the membrane thickness. This was confirmed through capacitance measurements on HEK-293 cells (Figure 4) performed both in dark and under visible light illumination. In dark, the membrane capacitance for not treated cells (CTRL) exhibits a value of $\sim 15.7 \pm 0.99 \mathrm{pF}$, while we observed a similar value $(16.6 \pm 0.65$ and $17.0 \pm 1.09 \mathrm{pF})$ following internalization of the two cross molecules. Conversely, the single-substituted and bisubstituted aze-pyridinium compounds (Az-1Pyr and Az-2Pyr) lead to an increase in the capacitance to $18.4 \pm 0.68$ and $18.2 \pm$ $1.12 \mathrm{pF}$, respectively, which can be ascribed to the abovementioned bilayer thinning effect. Note that although the 

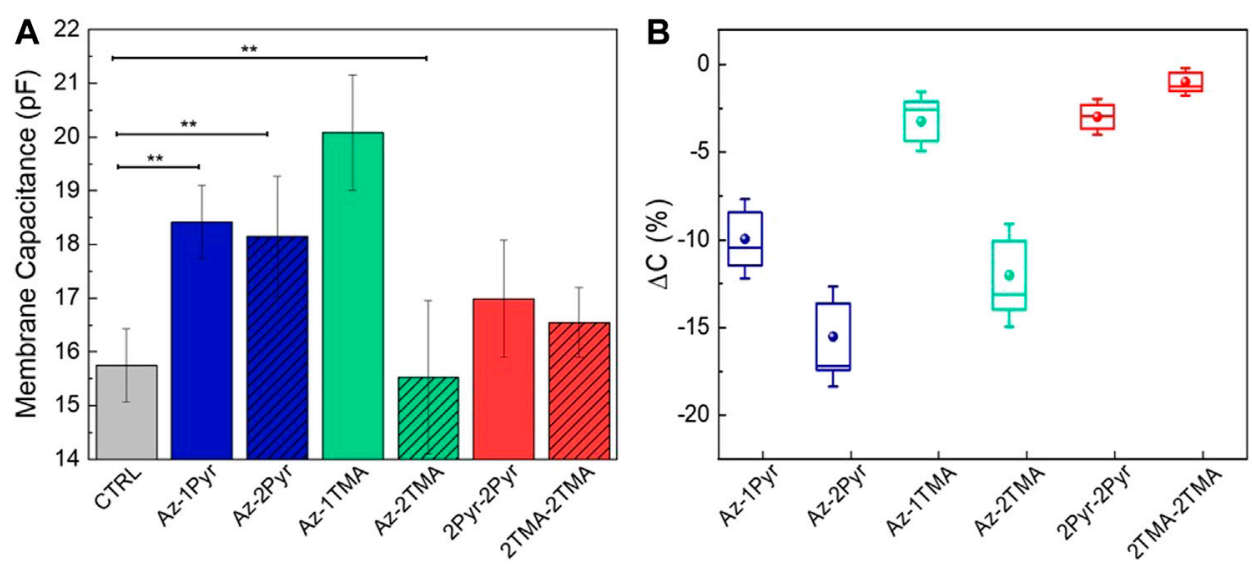

FIGURE 4 | Cell membrane capacitance data. (A) Dependency from the solely azobenzene molecules internalization into HEK-293 cells, and data are presented as mean \pm standard error of the mean $(n \geq 10)$. (B) Change in capacitance upon samples irradiation $(n \geq 10)$. Data are normalized to the amount of molecules partitioned in the cell membrane.

capacitance values reach the highest value with Az-1TMA (20 \pm $1.07 \mathrm{pF}$ ), this molecule showed instability problems between different runs of the same experiment, affecting the reliability of the results, as already mentioned. The similar value of membrane capacitance variation between $2 \mathrm{Pyr}-2 \mathrm{Pyr}$ and 2TMA-2TMA may be a hint of their ability to span the whole membrane, indicating that an alike reduction of the membrane thickness is applied (Figure 4A). With the measurements under illumination (light pulses duration $=200 \mathrm{~ms}$ ), we observed a marked decrease in the membrane capacitance for Az-1Pyr (17.72 pF) and Az-2Pyr (17.75 pF), most likely due to the partial restoration of the bilayer thickness (DiFrancesco et al., 2020). In general, we notice that for both Pyr- and TMAterminated molecules, the higher is the number of substituents, the smaller is the capacitance variation due to light stimulation. In addition, even though $2 \mathrm{Pyr}-2 \mathrm{Pyr}$ do not show any isomerization hint and $\mathrm{V}_{\mathrm{m}}$ modulation effect, it does induce a small capacitance change, probably owing to the membrane perturbation that do not achieve a second stationary state after the light pulse (Supplementary Figure S8). These capacitance changes could assume even more significance if normalized to the amount of molecules partitioned in the cell membrane. We worked out a correction parameter performing cell association experiments (Supplementary Figure S9). Specifically, the amount of molecules associated with cells was estimated measuring the decrease in the absorbance of a fixed amount of solution before and after $1 \mathrm{~h}$ of incubation with HEK-293 cells. The normalized capacitance variation under illumination (Figure 4B) corroborates the picture described above highlighting this time almost inappreciable changes induced by the cross molecules. Both membrane potential and capacitance experiments suggest a weaker effect applied by the ammoniumterminated molecules. Keeping in mind that the TMA group has a stronger interaction with the polar heads of the phospholipidic bilayer and the speculation of membrane thinning caused by dimers spanning the membrane, we assume that the ammonium- substituted molecules are much more bonded to the polar head of the membrane phospholipids, resulting in a weaker dimer formation. This interaction produces a smaller thinning of the membrane and subsequent lower changes in capacitance both in dark condition and during light excitation. In this scenario, the presence of the Pyr group makes the dimerization more efficient equalizing the interaction between two molecules and with the polar head of the phospholipids enhancing the photomechanical variation of the membrane thickness. This speculation, indeed, is in agreement with both the confocal measurement and collective isomerization dynamic, explaining the differences in the extracted time constants.

\section{CONCLUSION}

To summarize, six amphiphilic azobenzenes were synthesized as possible optical switches for photo-stimulation. We found that the different polar end groups and number of substituents attached to the central azobenzene unit have a profound influence on both optical and biological properties. In particular, monosubstituted and bisubstituted compounds reflect an intrinsic affinity with the phospholipid environment in solution (POPC), characterized by small red-shifts in their absorbance and PL spectra and a good photoisomerization efficiency when irradiated with light at the proper wavelength. However, photoreaction time constants of the aze-ammoniumterminated compounds are faster, suggesting the formation of weaker dimers, easier to break, when internalized into the membrane. Another hypothesis is that the higher polarity of the TMA termination intensifies their interaction with the phospholipid heads of the membrane bilayer. This is reflected in a weaker dimer and a subsequent less marked electrophysiological effect in real membranes $\mathrm{V}_{\mathrm{m}}$ modulation of HEK-293 cells and in a weaker ability to affect the membrane capacitance, both upon solely internalization into cells and upon internalization and subsequent light-induced isomerization. On 
the other hand, the two cross molecules studied appear to be the less effective in both measurements, likely due to the formation of intramembrane aggregates as suggested by their greatly redshifted photoluminescence spectra and their lack of isomerization in POPC liposomes mimicking the membrane environment.

In conclusion, we found that the membrane potential modulation originates from a convolution of a series of molecule-dependent properties (such as molecular symmetry, bulkiness, and number of substituents). Specifically, we found that although symmetric tetrasubstituted molecules exhibit a relatively high affinity for the membrane, they likely form nonisomerizing intramembrane aggregates. Conversely, the presence of the TMA cationic group likely leads to a less prominent mechanical membrane perturbation rather than the Pyr derivatives. We attribute this to a more effective dimerization of the pyridinium-based molecule which triggers a more evident thickness/capacitance photomodulation than the TMA groups. The optimization of such actuators could lead to the development of new candidates for achieving effective photomodulation of cells signaling, contributing to the progress of biosensors, biophotonics, biomedicine, and opto-neuroscience.

\section{MATERIALS AND METHODS}

\section{Chemicals}

DMSO (dimethyl sulfoxide) was purchased from Sigma-Aldrich (United Kingdom). FBS (fetal bovine serum) was obtained from Gibco (United Kingdom). POPC was purchased from Avanti Polar Lipids (Alabaster, AL), dissolved in chloroform, and stored at $-20{ }^{\circ} \mathrm{C}$. CellMask ${ }^{\mathrm{TM}}$ Deep Red was purchased from Invitrogen and CellBrite ${ }^{\mathrm{TM}}$ from Biotium, both used in accordance with the manufacturers' protocols.

For synthesis, unless otherwise stated, all chemicals and solvent were commercially available and used without further purification.

\section{Compounds Design and Synthesis}

The synthetic route for all the investigated compounds consists in the reduction of the nitro group of the Disperse Orange 3 dye into a primary amine, which is then alkylated with a, $\omega$-dibromohexane and finally treated with pyridine or trimethylamine (Supplementary Figure S10). The alkylation leads to the formation of a number of intermediates differently substituted with one, two, or four $\omega$-bromo-terminated alkyl chains. Among these compounds, we selected $\mathrm{Az}-1 \mathrm{Br}, \mathrm{Az}-2 \mathrm{Br}$, and $2 \mathrm{Br}-2 \mathrm{Br}$ which are then $\omega$-substituted with cationic groups, pyridinium or trimethylammonium.

Thin-layer chromatography was performed using silica gel on aluminum foil (Sigma Aldrich); ${ }^{1} \mathrm{H}$ and ${ }^{13} \mathrm{C}$ NMR spectra were collected with a Bruker ARX400. Mass spectroscopy was carried out with a Bruker Esquire 3000 plus.

\section{Synthesis of $\mathrm{Az}-1 \mathrm{Br}, \mathrm{Az}-2 \mathrm{Br}$, and $2 \mathrm{Br}-2 \mathrm{Br}$}

$0.69 \mathrm{~g}(3.2 \mathrm{mmol})$ of 1 (see Supplementary Figure S10) was mixed with $0.9 \mathrm{~g}(6.5 \mathrm{mmol})$ of $\mathrm{K}_{2} \mathrm{CO}_{3}$ and subjected to three
vacuum-Ar cycles and then stirred with $30 \mathrm{ml}$ of degassed acetonitrile. Subsequently, $0.6 \mathrm{ml} \quad(3.9 \mathrm{mmol})$ of $1,6-$ dibromohexane was added dropwise, and the reaction mixture was allowed to react under Ar atmosphere at $80{ }^{\circ} \mathrm{C}$ for 6 days in order to allow for the formation of azepane. A further addition of three times the amount of 1,6-dibromohexane was done, and the reaction mixture was left stirring for $120 \mathrm{~h}$ in the same conditions. The raw product was purified by flash chromatography using hexane as eluent. All the separated fractions were washed with portions of hexane and kept under vacuum in order to remove any excess of 1,6-dibromohexane. $41 \mathrm{mg}$ of $\mathrm{Az}-1 \mathrm{Br}$ (2.8\% yield), $42 \mathrm{mg}$ of $\mathrm{Az}-2 \mathrm{Br}$ (2.1\% yield), and $67 \mathrm{mg}$ of $2 \mathrm{Br}-2 \mathrm{Br}$ (2.4\% yield) were recovered.

\section{$\mathrm{Az}-1 \mathrm{Br}$}

${ }^{1} \mathrm{H}$ NMR: (400 MHz, DMSO) $\delta 7.63(2 \mathrm{H}, \mathrm{Ph}-\mathrm{H}, \mathrm{d}, \mathrm{J}=8.80 \mathrm{~Hz})$, $7.58(2 \mathrm{H}, \mathrm{Ph}-\mathrm{H}, \mathrm{d}, \mathrm{J}=8.80 \mathrm{~Hz}), 6.77(2 \mathrm{H}, \mathrm{Ph}-\mathrm{H}, \mathrm{d}, \mathrm{J}=8.80 \mathrm{~Hz})$, $6.64(2 \mathrm{H}, \mathrm{Ph}-\mathrm{H}, \mathrm{d}, \mathrm{J}=8.80 \mathrm{~Hz}), 6.21(1 \mathrm{H},-\mathrm{NH}, \mathrm{t}), 3.55(4 \mathrm{H}$, $\left.\mathrm{N}-\mathrm{CH}_{2^{-}}, \mathrm{t}\right), 3.35\left(2 \mathrm{H},-\mathrm{CH}_{2}-\mathrm{Br}, \mathrm{t}\right), 3.09\left(2 \mathrm{H},-\mathrm{CH}_{2}-\mathrm{NH}, \mathrm{m}\right)$, $1.83-1.42\left(16 \mathrm{H},-\mathrm{CH}_{2}, \mathrm{~m}\right)$;

\section{$\mathrm{Az}-2 \mathrm{Br}$}

${ }^{1} \mathrm{H}$ NMR: (400 MHz, DMSO) $\delta 7.63(4 \mathrm{H}, \mathrm{Ph}-\mathrm{H}, \mathrm{d}, \mathrm{J}=8.80 \mathrm{~Hz})$, $6.77(2 \mathrm{H}, \mathrm{Ph}-\mathrm{H}, \mathrm{d}, \mathrm{J}=8.80 \mathrm{~Hz}), 6.72(2 \mathrm{H}, \mathrm{Ph}-\mathrm{H}, \mathrm{d}, \mathrm{J}=8.80 \mathrm{~Hz})$, $3.55\left(8 \mathrm{H},-\mathrm{N}-\mathrm{CH}_{2}, \mathrm{t}\right), 3.35\left(2 \mathrm{H},-\mathrm{CH}_{2}-\mathrm{Br}, \mathrm{t}\right), 1.82-1.35(24 \mathrm{H}$, $\left.-\mathrm{CH}_{2}, \mathrm{~m}\right)$;

\section{Br-2Br}

${ }^{1} \mathrm{H}$ NMR: (400 MHz, DMSO) $\delta 7.62(4 \mathrm{H}, \mathrm{Ph}-\mathrm{H}, \mathrm{d}, \mathrm{J}=8.80 \mathrm{~Hz})$, $6.72(4 \mathrm{H}, \mathrm{Ph}-\mathrm{H}, \mathrm{d}, \mathrm{J}=8.80 \mathrm{~Hz}), 3.55\left(8 \mathrm{H}, \mathrm{N}-\mathrm{CH}_{2^{-}}, \mathrm{t}\right), 3.35(8 \mathrm{H}$, $\left.-\mathrm{CH}_{2}-\mathrm{Br}, \mathrm{t}\right), 1.82(8 \mathrm{H}, \mathrm{m}), 1.56\left(8 \mathrm{H},-\mathrm{CH}_{2}, \mathrm{~m}\right), 1.44\left(8 \mathrm{H},-\mathrm{CH}_{2}\right.$, $\mathrm{m}), 1.35\left(8 \mathrm{H},-\mathrm{CH}_{2}, \mathrm{~m}\right)$; synthesis of Az-1Pyr, Az-2Pyr, Az1TMA, Az-2TMA, 2Pyr-2Py, and 2TMA-2TMA.

As a general procedure, the $\omega$-bromoalkyl chains of precursors above reported were then functionalized with pyridine or trimethylamine. The specific reactions are detailed as follows.

Az-1Pyr: $0.028 \mathrm{~g}(0.06 \mathrm{mmol})$ of Az-1Br was dissolved in $8 \mathrm{ml}$ of pyridine and left stirring for $48 \mathrm{~h}$ at room temperature. We obtained the desired product in quantitative yield, which was then washed with small portions of hexane and dried under vacuum $\left(10^{-1} \mathrm{mbar}, 60{ }^{\circ} \mathrm{C}\right) .{ }^{1} \mathrm{H}-\mathrm{NMR}(400 \mathrm{MHz}, \mathrm{DMSO}-\mathrm{d} 6) \delta 9.12(2 \mathrm{H}$, Pyr-H, d), 8.62 (1H, Pyr-H, t), 8.13 (2H, Pyr-H, t), 7.63 (4H, Ph$\mathrm{H}, \mathrm{d}, \mathrm{J}=9.0 \mathrm{~Hz}), 6.78(4 \mathrm{H}, \mathrm{Ph}-\mathrm{H}, \mathrm{d}, \mathrm{J}=9.17 \mathrm{~Hz}), 6.26(1 \mathrm{H}, \mathrm{NH}, \mathrm{s})$, $4.61\left(2 \mathrm{H},-\mathrm{CH}_{2}-\mathrm{Pyr}, \mathrm{t}\right), 3.55\left(4 \mathrm{H},-\mathrm{CH}_{2}-\mathrm{N}, \mathrm{t}\right), 1,94-1.35(16 \mathrm{H}, \mathrm{m})$.

$\mathrm{Az}-2 \mathrm{Pyr}$ was synthesized by dissolving $0.022 \mathrm{~g}(0.035 \mathrm{mmol})$ of $\mathrm{Az}-2 \mathrm{Br}$ in $6 \mathrm{ml}$ of pyridine and left stirring for $48 \mathrm{~h}$ at room temperature. We obtained the desired product in quantitative yield, which was washed with small portions of hexane and dried under vacuum $\left(10^{-1} \mathrm{mbar}, 60^{\circ} \mathrm{C}\right) .{ }^{1} \mathrm{H}-\mathrm{NMR}$ (400 MHz, DMSO) $\delta$ 9.09 (4H, Pyr-H, d), 8.63 (2H, Pyr-H, t), 8.17 (4H, Pyr-H, t), 7.65 $(4 \mathrm{H}, \mathrm{Ph}-\mathrm{H}, \mathrm{d}, \mathrm{J}=9.0 \mathrm{~Hz}), 6.8(2 \mathrm{H}, \mathrm{Ph}-\mathrm{H}, \mathrm{d}, \mathrm{J}=9.2 \mathrm{~Hz}), 6.72(2 \mathrm{H}$, $\mathrm{Ph}-\mathrm{H}, \mathrm{d}, \mathrm{J}=9.2 \mathrm{~Hz}), 4.59\left(4 \mathrm{H},-\mathrm{CH}_{2}-\mathrm{Pyr}, \mathrm{t}\right), 3.55\left(4 \mathrm{H},-\mathrm{CH}_{2}-\mathrm{N}, \mathrm{t}\right)$, $1.94-1.33(24 \mathrm{H}, \mathrm{m})$.

Az-1TMA: $0.028 \mathrm{~g}(0.06 \mathrm{mmol})$ of $\mathrm{Az}-1 \mathrm{Br}$ was dissolved in $4 \mathrm{ml}$ of ethanol adding dropwise $0.3 \mathrm{ml}$ of trimethylamine and left stirring for $48 \mathrm{~h}$ at $80{ }^{\circ} \mathrm{C}$. We obtained the desired product in quantitative yield, which was then washed with small portion of 
hexane and dried under vacuum $\left(10^{-1} \mathrm{mbar}\right) .{ }^{1} \mathrm{H}-\mathrm{NMR}$ $(400 \mathrm{MHz}, \mathrm{DMSO}) \delta 7.64(4 \mathrm{H}, \mathrm{Ph}-\mathrm{H}, \mathrm{d}, \mathrm{J}=9.05 \mathrm{~Hz}), 6.77$ $(2 \mathrm{H}, \mathrm{Ph}-\mathrm{H}, \mathrm{d}, \mathrm{J}=9.17 \mathrm{~Hz}), 6.64(2 \mathrm{H}, \mathrm{Ph}-\mathrm{H}, \mathrm{d}, \mathrm{J}=9.17 \mathrm{~Hz})$, $6.30(1 \mathrm{H}, \mathrm{NH}, \mathrm{s}), 3.54\left(4 \mathrm{H},-\mathrm{CH}_{2}-\mathrm{N}, \mathrm{t}\right), 3.05(9 \mathrm{H}, \mathrm{s}, \mathrm{TMA}-\mathrm{CH} 3)$, $1.76-1.35(16 \mathrm{H}, \mathrm{m})$.

Az-2TMA was synthesized by dissolving $0.07 \mathrm{~g}(0.11 \mathrm{mmol})$ of $\mathrm{Az}-2 \mathrm{Br}$ in $4 \mathrm{ml}$ of ethanol and dropwise adding $0.6 \mathrm{ml}$ of trimethylamine. The reaction was left stirring for $48 \mathrm{~h}$ at $80{ }^{\circ} \mathrm{C}$. We obtained the desired product in quantitative yield, which was then washed with small portion of hexane and dried under vacuum $\left(10^{-1} \mathrm{mbar}\right)$ giving ${ }^{1} \mathrm{H}-\mathrm{NMR}$ (400 MHz, DMSO) $\delta: 7.64(2 \mathrm{H}, \mathrm{Ph}-\mathrm{H}, \mathrm{d}, \mathrm{J}=9.05 \mathrm{~Hz}), 7.59(2 \mathrm{H}, \mathrm{Ph}-\mathrm{H}, \mathrm{d}, \mathrm{J}=8.80 \mathrm{~Hz})$, $6.79(2 \mathrm{H}, \mathrm{Ph}-\mathrm{H}, \mathrm{d}, \mathrm{J}=9.17 \mathrm{~Hz}), 6.65(2 \mathrm{H}, \mathrm{Ph}-\mathrm{H}, \mathrm{d}, \mathrm{J}=8.92 \mathrm{~Hz})$, $3.54\left(4 \mathrm{H},-\mathrm{CH}_{2}-\mathrm{N}, \mathrm{t}\right), 3.04(18 \mathrm{H}, \mathrm{TMA}-\mathrm{CH} 3, \mathrm{~s}), 1.8-1.4(\mathrm{~m}, 24 \mathrm{H})$.

2Pyr-2Pyr: $0.050 \mathrm{~g}(0.058 \mathrm{mmol}) 2 \mathrm{Br}-2 \mathrm{Br}$ is dissolved in $5 \mathrm{ml}$ of pyridine and left stirring for $120 \mathrm{~h}$ at room temperature. We obtained the desired product in quantitative yield, which was then washed with small portions of hexane and dried under vacuum $\left(10^{-1} \mathrm{mbar}, 60{ }^{\circ} \mathrm{C}\right) .{ }^{1} \mathrm{H}-\mathrm{NMR}(400 \mathrm{MHz}, \mathrm{DMSO}) \delta 9.09(8 \mathrm{H}, \mathrm{Pyr}-$ H, d), 8.63 (4H, Pyr-H, t), 8.17 (8H, Pyr-H, d), 7.65 (4H, Ph-H, d, $\mathrm{J}=9.0 \mathrm{~Hz}), 6.7(4 \mathrm{H}, \mathrm{Ph}-\mathrm{H}, \mathrm{d}, \mathrm{J}=9.2 \mathrm{~Hz}), 4.62\left(8 \mathrm{H},-\mathrm{CH}_{2}-\mathrm{Pyr}, \mathrm{t}\right)$, $1.94-1.35(32 \mathrm{H}, \mathrm{m})$.

2TMA-2TMA: $0.052 \mathrm{~g}(0.06 \mathrm{mmol})$ of $2 \mathrm{Br}-2 \mathrm{Br}$ was stirred in $4 \mathrm{ml}$ of ethanol; $0.6 \mathrm{ml}$ of trimethylamine solution is then added dropwise and left stirring for $48 \mathrm{~h}$ at $80{ }^{\circ} \mathrm{C}$. We obtained the desired product in quantitative yield, which was then washed with small portion of hexane and dried under vacuum $\left(10^{-1} \mathrm{mbar}\right.$, $\left.60{ }^{\circ} \mathrm{C}\right) .{ }^{1} \mathrm{H}-\mathrm{NMR}(400 \mathrm{MHz}, \mathrm{DMSO}) \delta 7.64(4 \mathrm{H}, \mathrm{Ph}-\mathrm{H}, \mathrm{d}, \mathrm{J}=$ $9.1 \mathrm{~Hz}), 6.74(4 \mathrm{H}, \mathrm{Ph}-\mathrm{H}, \mathrm{d}, \mathrm{J}=8.9 \mathrm{~Hz}), 3.05(36 \mathrm{H}, \mathrm{TMA}-\mathrm{CH} 3, \mathrm{~s})$, 1.69-1.34 (32H, m).

For all the measurements in water or POPC, molecules were dissolved into deionized water. From this water solution, further internalization into artificial membranes was carried out, avoiding the preparation of a DMSO stock solution so as to exclude the presence of DMSO microdomains which could have affected plasma membrane properties and influenced the molecules affinity for the membrane environment.

\section{UV-Vis Absorption and Photoluminescence Measurements}

For the UV-Vis absorption measurements, a Perkin Elmer Lambda 1050 spectrophotometer was employed, with deuterium (180-320 nm) and tungsten (320-3300 nm) lamps and three detectors (photomultiplier 180-860 nm, InGaAs 860-1300 nm, and PbS 1300-3300 nm). Absorption spectra were normalized according to a reference spectrum taken at $100 \%$ transmission (without the sample), $0 \%$ transmission (with an internal shutter), and in the presence of the reference solvent. PL measurements were performed with an iHR320 Horiba NanoLog Fluorometer equipped with a xenon lamp, two monochromators, and two detectors (photomultiplier and InGaAs). Dynamic UV-Vis absorption measurements were performed employing a fibre-fed spectrometer (Avantes, AvaSpec-HS2048XL-EVO) equipped with a deuterium-halogen lamp (AvaLight- $\mathrm{D}(\mathrm{H})-\mathrm{S})$. To induce the isomerization process, an LED lamp (Thorlabs M470L3-C5) centered at $470 \mathrm{~nm}$ with a
$100 \mathrm{~mW}$ power was used and placed on top of a quartz cuvette (1 mm path) containing the molecules solutions. The sample was illuminated uniformly.

\section{Liposome Synthesis}

Liposomes were prepared through standard extrusion procedure. Lipids in chloroform solution mixed with methanol (1:1) were dried out under high vacuum with the use of a rotary evaporator, and the resulting thin lipid film was kept overnight at $-20^{\circ} \mathrm{C}$. The film was then hydrated with a buffer solution $(10 \mathrm{mM}$ Tris, $100 \mathrm{mM} \mathrm{NaCl}, \mathrm{pH} \mathrm{7.4)}$ and subjected to freeze-thaw cycles and extrusions through a polycarbonate membrane (pore size $100 \mathrm{~nm}$ ) at room temperature (above POPC $\mathrm{T}_{\mathrm{m}}$ ). The samples were kept at $4{ }^{\circ} \mathrm{C}$, and liposome concentration in the buffer suspension was $5 \mathrm{mg} / \mathrm{ml}$. The vesicles were used within the week of preparation. Molecule intercalation was performed by simply addition of the composites to the liposome suspension and vortexing the solution.

\section{Cell Culture Maintenance}

Both confocal imaging and electrophysiology in vitro procedures were performed using the immortalized cell line HEK-293 (Human Embryonic Kidney), purchased from ATCC. HEK293 cells were cultured in T-75 cell culture flasks containing Dulbecco's Modified Eagle Medium high glucose (DMEM-HG) culture medium, supplemented with $10 \%$ heat inactivated FBS, $2 \%$ penicillin/streptomycin, and $1 \%$ L-glutamine. Culture flasks were maintained in a humidified incubator at $37^{\circ} \mathrm{C}$ with $5 \% \mathrm{CO}_{2}$. When at confluence, cells were enzymatically detached from the flasks with a $1 \mathrm{x}$ trypsin-EDTA solution, plated on sterilized glass coverslips and left to grow. Prior to cell plating, a layer of fibronectin $\left(2 \mu \mathrm{g} \mathrm{ml}^{-1}\right.$ in PBS buffer solution) was deposited on the sample surface and incubated for $1 \mathrm{~h}$ at $37^{\circ} \mathrm{C}$, to promote cellular adhesion.

\section{Confocal Imaging}

For confocal imaging, each molecule was incubated separately at a concentration of $25 \mu \mathrm{M}$ (from a $2 \mathrm{mM}$ stock solution) in the glass-plated cells samples, prepared as described in the cell culture section (see above). Molecules were incubated for $7 \mathrm{~min}$ at $37^{\circ} \mathrm{C}$, in a humidified $5 \% \mathrm{CO}_{2}$ atmosphere. After incubation, samples were repeatedly washed with PBS in order to remove cellunbound molecules. Cell membranes were then uniformly labeled with CellMask ${ }^{\mathrm{TM}}$ Deep Red (red dye) or CellBrite (blue dye) depending on the different molecule emission range in order to avoid spectral overlap. Coverslips with cells were washed with warmed PBS and mounted on an inverted confocal laser scanning microscope Nikon Eclipse $\mathrm{Ti}^{2}$ (Nikon Instruments). Live-cell $\mathrm{Z}$-series stack acquisition was performed using an Olympus $60 \mathrm{X}$ oil objective. Confocal images were analyzed with Fiji's JACoP plugin to evaluate the colocalization of the azobenzene molecules with the cell membrane. Colocalization analysis was performed using the Fiji plugins JACoP.

\section{Cell Association Experiments}

Culture medium (DMEM-HG) of glass-seeded HEK-293 cell samples (confluency 80\%) was removed and substituted with 
PBS. Samples were stained in clear 12-well plates with each azobenzene molecules [25 $\mu \mathrm{M}$ in PBS] and maintained at $37^{\circ} \mathrm{C}$ for $60 \mathrm{~min}$ in dark. $200 \mu \mathrm{l}$ of each supernatant was transferred to a clean 96-well plate for UV-Vis absorption with a Tecan Spark10M plate reader. Absorbance was measured at $470 \mathrm{~nm}$ for all the molecules. Control samples with no cells were treated the same, and their absorbance values represented the total azomolecules from which the supernatant values were subtracted to give the amount associated with cells. All conditions and controls were measured in triplicate.

\section{Electrophysiology}

Standard patch clamp recordings were performed with an Axopatch 200 B (Axon Instruments) coupled with a Nikon Eclipse Ti inverted microscope. Glass-seeded HEK-293 cells were measured in whole-cell configuration with freshly pulled glass pipettes (4-7 M $\Omega$ ), filled with the following intracellular solution [mM]: $12 \mathrm{KCl}, 125 \mathrm{~K}$-Gluconate, $1 \mathrm{MgCl}_{2}, 0.1 \mathrm{CaCl}_{2}, 10$ EGTA, 10HEPES, and 10 ATP-Na2. The extracellular solution contained [mM] $135 \mathrm{NaCl}, 5.4 \mathrm{KCl}$, 5HEPES, 10Glucose, 1.8 $\mathrm{CaCl}_{2}$, and $1 \mathrm{MgCl}_{2}$. Only single cells were selected for recordings. Acquisition was performed with pClamp-10 software (Axon Instruments). Membrane currents were low pass filtered at $2 \mathrm{kHz}$ and digitized with a sampling rate of $10 \mathrm{kHz}$ (Digidata 1440 A, Molecular Devices). A cyan LED coupled to the fluorescence port of the microscope and characterized by maximum emission wavelength at $470 \mathrm{~nm}$ provided the excitation light source. The illuminated spot on the sample has an area of $0.23 \mathrm{~mm}^{2}$ and a photoexcitation density of $50 \mathrm{~mW} /$ $\mathrm{mm}^{2}$, as measured at the output of the microscope objective (Pobj). Data were analyzed with Origin 9.0 (OriginLab Corporation) and with MATLAB software.

\section{Capacitance Measurements}

A double sinusoidal voltage-clamp signal was applied to the cell in whole-cell configuration. The response current signal was acquired and membrane capacitance, membrane resistance, and access resistance are then extracted fitting the current with a custom MATLAB program.

\section{REFERENCES}

Bandara, H. M., and Burdette, S. C. (2012). Photoisomerization in different classes of azobenzene. Chem. Soc. Rev. 41, 1809-1825. doi:10.1039/c1cs15179g

Bareket-Keren, L., and Hanein, Y. (2014). Novel interfaces for light directed neuronal stimulation: advances and challenges. Int. J. Nanomed. 9 Suppl 1, 65-83. doi:10.2147/IJN.S51193

Beharry, A. A., and Woolley, G. A. (2011). Azobenzene photoswitches for biomolecules. Chem. Soc. Rev. 40, 4422-4437.doi:10.1039/c1cs15023e

Bolte, S., and Cordelières, F. P. (2006). A guided tour into subcellular colocalization analysis in light microscopy. J. Microsc. 224, 213-232. doi:10.1111/j.1365-2818. 2006.01706.x

Chae, M. S., Yang, Z., and Liu, W. (2010). Implantable neural prostheses 2: techniques and engineering approaches. Available at: http://www.springerlink. com/index/10.1007/978-0-387-98120-8.

Crecca, C. R., and Roitberg, A. E. (2006). Theoretical study of the isomerization mechanism of azobenzene and disubstituted azobenzene derivatives. J. Phys. Chem. 110, 8188-8203. doi:10.1021/jp057413c

\section{Statistical Analysis}

Statistical significance between two conditions was evaluated using Student's t-test. In all the reported data, ${ }^{*} p<0.05,{ }^{* *} p<0.01$, and ${ }^{* * *} p<0.001$. Statistical analysis was performed using Excel.

\section{DATA AVAILABILITY STATEMENT}

The original contributions presented in the study are included in the article/Supplementary Material; further inquiries can be directed to the corresponding authors.

\section{AUTHOR CONTRIBUTIONS}

GB and VV contributed equally to this work. CB designed and engineered the molecules. VS performed the synthesis of the molecules. GB performed the spectroscopic characterization, synthesized the liposomes, and performed cell association experiments. VV and FL performed the in vitro patch-clamp experiments and analyzed the data. VV performed the confocal imaging and capacitance measurements. GL and CB conceived the work. GP contributed to perform spectroscopic characterization, data analysis, and paper writing. GB and VV wrote the manuscript.

\section{FUNDING}

GP thanks Fondazione Cariplo (grant no. 2018-0979) for the financial support. GL thanks Fondazione Cariplo (project Nanosparks, grant no 2018-0505) for the financial support.

\section{SUPPLEMENTARY MATERIAL}

The Supplementary Material for this article can be found online at: https://www.frontiersin.org/articles/10.3389/fmats.2020.631567/ full\#supplementary-material.

Di Maria, F., Lodola, F., Zucchetti, E., Benfenati, F., and Lanzani, G. (2018). The evolution of artificial light actuators in living systems: from planar to nanostructured interfaces. Chem. Soc. Rev. 47, 4757-4780. doi:10.1039/ C7CS00860K

DiFrancesco, M. L., Lodola, F., Colombo, E., Maragliano, L., Bramini, M., Paternò, G. M., et al. (2020). Neuronal firing modulation by a membranetargeted photoswitch. Nat. Nanotechnol. 15, 296-306. doi:10.1038/s41565019-0632-6

Fang, Y., Meng, L., Prominski, A., Schaumann, E. N., Seebald, M., and Tian, B. (2020). Recent advances in bioelectronics chemistry. Chem. Soc. Rev. 49, 7978-8035. doi:10.1039/D0CS00333F

Fenno, L., Yizhar, O., and Deisseroth, K. (2011). The development and application of optogenetics. Annu. Rev. Neurosci. 34, 389-412. doi:10.1146/annurev-neuro061010-113817

Fuchter, M. J. (2020). On the promise of photopharmacology using photoswitches: a medicinal chemist's perspective. J. Med. Chem. 20, 11436-11447. doi:10.1021/ acs.jmedchem.0c00629

Geng, S., Wang, Y., Wang, L., Kouyama, T., Gotoh, T., Wada, S., et al. (2017). A light-responsive self-assembly formed by a cationic azobenzene derivative and 
SDS as a drug delivery system. Sci. Rep. 7, 39202-39206. doi:10.1038/ srep39202

Griffiths, J. (1972). II. Photochemistry of azobenzene and its derivatives. Chem. Soc. Rev. 1, 481-493. doi:10.1039/CS9720100481

Hodgkin, A. L., and Huxley, A. F. (1952). A quantitative description of membrane current and its application to conduction and excitation in nerve. J. Physiol. 117, 500-544. doi:10.1113/jphysiol.1952.sp004764

Hopkins, J., Travaglini, L., Lauto, A., Cramer, T., Fraboni, B., Seidel, J., et al. (2019). Photoactive organic substrates for cell stimulation: progress and perspectives. Adv. Mater. Technol. 4, 1-10. doi:10.1002/admt.201800744

Paternò, G. M., Bondelli, G., Sakai, V. G., Sesti, V., Bertarelli, C., and Lanzani, G. (2020a). The effect of an intramembrane light-actuator on the dynamics of phospholipids in model membranes and intact cells. Langmuir. 39, 11517-11527. doi:10.1021/acs.langmuir.0c01846

Paternò, G. M., Colombo, E., Vurro, V., Lodola, F., Cimò, S., Sesti, V., et al. (2020b). Membrane environment enables ultrafast isomerization of amphiphilic azobenzene. Adv. Sci. 7, 1903241. doi:10.1002/advs.201903241

Russew, M. M., and Hecht, S. (2010). Photoswitches: from molecules to materials. Adv Mater Weinheim. 22, 3348-3360. doi:10.1002/adma.200904102

Scanziani, M., and Häusser, M. (2009). Electrophysiology in the age of light, 461. doi:10.1038/nature08540
Tochitsky, I., Helft, Z., Meseguer, V., Fletcher, R. B., Vessey, K. A., Telias, M., et al. (2016). How azobenzene photoswitches restore visual responses to the blind retina. Neuron. 92, 100-113. doi:10.1016/j.neuron.2016.08.038

Uchida, K. (2004). Photochromism. Molecules and systems. Edited by heinz dürr and henri bouas-laurent. Angew. Chem. Int. Ed. 43, 3362. doi:10.1002/anie. 200385129

Velema, W. A., Szymanski, W., and Feringa, B. L. (2014). Photopharmacology: beyond proof of principle. J. Am. Chem. Soc. 136, 2178-2191. doi:10.1021/ ja413063e

Conflict of Interest: The authors declare that the research was conducted in the absence of any commercial or financial relationships that could be construed as a potential conflict of interest.

Copyright (c) 2021 Vurro, Bondelli, Sesti, Lodola, Paternò, Lanzani and Bertarelli. This is an open-access article distributed under the terms of the Creative Commons Attribution License (CC BY). The use, distribution or reproduction in other forums is permitted, provided the original author(s) and the copyright owner(s) are credited and that the original publication in this journal is cited, in accordance with accepted academic practice. No use, distribution or reproduction is permitted which does not comply with these terms. 